\title{
Chemotherapy versus Best Supportive Care in Advanced Biliary Tract Carcinoma: A Multi-institutional Propensity Score Matching Analysis
}

\author{
Jun Ho Ji, MD ${ }^{1}$ \\ Young Saing Kim, MD, PhD² \\ Inkeun Park, MD2 \\ Soon II Lee, MD, PhD \\ Rock Bum Kim, MD, PhD ${ }^{4}$ \\ Joon Oh Park, MD, PhD \\ Sung Yong Oh, MD, $\mathrm{PhD}{ }^{6}$ \\ In Gyu Hwang, MD, PhD \\ Joung-Soon Jang, MD, $\mathrm{PhD}^{7}$ \\ Haa-Na Song, MD ${ }^{8}$ \\ Jung-Hun Kang, MD, $P h D^{8}$
}

\begin{abstract}
${ }^{1}$ Division of Hematology-Oncology, Samsung Changwon Hospital, Sungkyunkwan University School of Medicine, Changwon, ${ }^{2}$ Division of Hematology and Oncology, Department of Internal Medicine, Gachon University Gil Medical Center, Incheon, ${ }^{3}$ Department of Internal Medicine, Dankook University College of Medicine, Cheonan, ${ }^{4}$ Regional Cardiocerebrovascular Center, Gyeongsang National University Hospital, Jinju, ${ }^{5}$ Division of Hematology-Oncology, Department of Medicine, Samsung Medical Center, Sungkyunkwan University School of Medicine, Seoul, ${ }^{6}$ Department of Internal Medicine, Dong-A University College of Medicine, Busan, ${ }^{7}$ Department of Internal Medicine, Chung-Ang University College of Medicine, Seoul, ${ }^{8}$ Department of Internal Medicine, Gyeongsang National University School of Medicine, Jinju, Korea
\end{abstract}

Correspondence: Jung-Hun Kang, MD, PhD Department of Internal Medicine, Gyeongsang National University College of Medicine, 79 Gangnam-ro, Jinju 52727, Korea Tel: 82-55-750-8063

Fax: 82-55-758-9122

E-mail: newatp@naver.com

Received January 24, 2017

Accepted August 1, 2017

Published Online August 23, 2017

\section{Purpose}

Although chemotherapy is recommended by various guidelines for advanced biliary tract cancer (BTC), the evidence supporting its use over best supportive care (BSC) is limited. The aim of this study was to investigate the survival benefit of chemotherapy over that of BSC in advanced BTC patients.

\section{Materials and Methods}

Advanced BTC patients with a good performance status (Eastern Cooperative Oncology Group [ECOG] 0-2) were eligible for the study. Data were retrospectively collected from four tertiary cancer centers and analyzed using propensity score matching (PSM). Of the 604 patients enrolled, 206 received BSC and 398 received chemotherapy. PSM analysis was performed using the following variables: age, ECOG status, carcinoembryonic antigen (CEA) level, white blood cell level, albumin level, total bilirubin level, and aspartate aminotransferase level. The sample size of each group was 164 patients after PSM. Median survival was compared between the two groups by using the Kaplan-Meier method, and prognostic factors were investigated using Cox proportional regression analysis.

\section{Results}

In post-PSM analysis, the respective median survival for the chemotherapy and BSC groups was dependent on the following prognostic factors: total population, 12.0 months vs. 7.5 months ( $p=0.001$ ); locally advanced disease, 16.7 months vs. 13.4 months ( $p=0.490)$; cancer antigen $19-9 \leq 100 \mathrm{IU} / \mathrm{mL}, 12.7$ months vs. 10.6 months $(p=0.330)$; and CEA $\leq 3.4$ $\mathrm{ng} / \mathrm{mL}, 17.1$ months vs. 10.6 months ( $p=0.052)$.

\section{Conclusion}

Chemotherapy improved overall survival of patients with advanced BTC who had a good performance status. However, this survival benefit was not observed in BTC patients with locally advanced disease or with lower tumor marker. Individualized approach is needed for initiation of palliative chemotherapy in advanced BTC. 


\section{Introduction}

Biliary tract cancer (BTC) is a heterogeneous subgroup of tumors, including intrahepatic cholangiocarcinoma, extrahepatic cholangiocarcinoma, gallbladder cancer, and ampulla of Vater cancer. BTC has a very poor prognosis. Therapeutic options for patients with BTC include surgery, photodynamic therapy, radiation therapy, liver transplantation, chemoradiation therapy, and chemotherapy. Complete resection could improve long-term survival, but the diagnosis of BTC is often challenging and as such, many cases are inoperable by the time of diagnosis. For patients with inoperable disease, gemcitabine in combination with cisplatin has been administered as the standard chemotherapy regimen. Prior to the establishment of a standard regimen, many chemotherapeutic agents were studied for their efficacy against advanced BTC, but the majority of studies have had limited value in establishing a standard regimen because of a small sample size or non-randomized design; therefore, there have been no promising results in randomized phase III studies.

To develop a standard chemotherapy regimen, a phase III clinical study of a chemotherapy drug with proven efficacy and safety must be conducted, comparing the outcomes of the chemotherapy drug versus those of best supportive care (BSC). In pancreatic cancer, 5-fluorouracil (5-FU)-based chemotherapy was shown to be superior over BSC in the late 1970s, and thereafter, the survival rates have improved stepwise with 5-FU and more effective drugs [1-3]. Recently, many studies for second-line chemotherapy treatment of advanced BTC have been published $[4,5]$. Most patients with advanced BTC had outcomes that were comparable with those reported in previous studies; however, frontline chemotherapy provides a survival benefit for many patients, even if it is not effective for everyone with advanced BTC. In fact, depending on the clinical situation, advanced BTC has considerable variation with respect to chemotherapy response and survival time. Therefore, a survival comparison between advanced BTC patients with the same conditions receiving chemotherapy or BSC should aid clinicians in identifying the appropriate candidates for palliative chemotherapy.

In our previous study [6], the natural history of inoperable BTC was only 7.1 months, but patients with low levels of tumor markers or locally advanced BTC had modest survival times (10.6 months and 13.8 months, respectively) without any treatment. This survival time was comparable to historical data from patients with advanced BTC who received chemotherapy. As an extension of our earlier study, we conducted a comparative analysis between chemotherapy and BSC in patients with advanced BTC, applying propensity score matching (PSM) to evaluate variables that were dispro- portionate between the groups.

\section{Materials and Methods}

\section{Patient populations}

\section{1) BSC group}

The data for the BSC group were collected at six cancer centers in Korea. We selected 206 patients who met the following criteria for inclusion in the study: (1) histologic diagnosis of locally advanced or metastatic BTC, or (2) Eastern Cooperative Oncology Group (ECOG) performance status of 0-2 (eligible to receive chemotherapy). Exclusion criteria were patients who had (1) undergone curative surgery, chemotherapy, and radiation therapy for primary cancer; (2) initially resectable disease; or (3) an ECOG performance status of 3-4. The data on this cohort were reported elsewhere in detail [6].

\section{2) Chemotherapy group}

Data for the chemotherapy group were collected at four cancer centers in Korea. We selected 398 patients who met the following criteria for inclusion in the study: (1) a histologic diagnosis of locally advanced or metastatic BTC, (2) administration of frontline chemotherapy, and (3) an ECOG performance status of 0-2. Patients who had (1) undergone curative surgery and radiation therapy for primary cancer, (2) initially resectable disease, or (3) an ECOG performance status of 3-4 were excluded from the study.

\section{Variables included in the study}

Baseline characteristics included age, sex, disease status (locally advanced or metastatic disease), ECOG performance status, frontline chemotherapy regimen, white blood cell (WBC) count, neutrophil/lymphocyte/monocyte counts, red cell distribution width, mean cell volume, hemoglobin level, platelet count, erythrocyte sedimentation rate, C-reactive protein, albumin, total bilirubin, alkaline phosphatase, aspartate aminotransferase (AST), alanine transaminase (ALT), carcinoembryonic antigen (CEA), and cancer antigen 19-9 (CA 19-9) levels, and whether or not biliary drainage was performed. The cut-off level of categorical variables was defined as follows: ECOG 0-1 and 2; CA 19-9 $\leq 100 \mathrm{IU} / \mathrm{mL}$ and $>100 \mathrm{IU} / \mathrm{mL} ; \mathrm{CEA} \leq 3.4 \mathrm{ng} / \mathrm{mL}$ and $>3.4 \mathrm{ng} / \mathrm{mL}$; WBC $<3,500$ cells $/ \mathrm{mm}^{3}, 3,500$ cells $/ \mathrm{mm}^{3} \leq \mathrm{WBC}<10,000$ cells $/$ $\mathrm{mm}^{3}$, and $\mathrm{WBC} \geq 10,000$ cells $/ \mathrm{mm}^{3}$. 
Table 1. Baseline characteristics of the patients

\begin{tabular}{|c|c|c|c|c|c|c|}
\hline \multirow[b]{2}{*}{ Characteristic } & \multicolumn{3}{|c|}{ Before propensity score matched } & \multicolumn{3}{|c|}{ After propensity score matched } \\
\hline & $\begin{array}{l}\text { Chemotherapy } \\
\qquad(n=398)\end{array}$ & $\begin{array}{l}\text { Natural history } \\
\quad(n=206)\end{array}$ & p-value & $\begin{array}{l}\text { Chemotherapy } \\
\qquad(\mathrm{n}=164)\end{array}$ & $\begin{array}{l}\text { Natural history } \\
(n=164)\end{array}$ & p-value \\
\hline \multicolumn{7}{|l|}{ Sex } \\
\hline Male & $226(56.8)$ & $115(55.8)$ & 0.863 & $93(56.7)$ & $91(55.5)$ & 0.911 \\
\hline Female & $172(43.2)$ & $91(44.2)$ & & $71(43.3)$ & $73(44.5)$ & \\
\hline Age & $60.7 \pm 10.0$ & $69.9 \pm 10.6$ & $<0.001$ & $67.4 \pm 6.7$ & $67.9 \pm 10.1$ & 0.592 \\
\hline \multicolumn{7}{|l|}{ Extent of disease } \\
\hline locally advanced & $110(27.7)$ & $42(22.7)$ & 0.224 & $29(17.7)$ & $36(22.0)$ & 0.406 \\
\hline Metastatic & $287(72.3)$ & $143(77.3)$ & & $135(82.3)$ & $128(78.0)$ & \\
\hline \multicolumn{7}{|l|}{ ECOG } \\
\hline 0 or 1 & $346(86.9)$ & $125(60.7)$ & $<0.001$ & $116(70.7)$ & $109(66.5)$ & 0.475 \\
\hline 2 & $52(13.1)$ & $81(39.3)$ & & $48(29.3)$ & $55(33.5)$ & \\
\hline \multicolumn{7}{|l|}{ CA 19-9 (IU/mL) } \\
\hline$\leq 100$ & $175(48.9)$ & $84(43.5)$ & 0.245 & $63(42.3)$ & $67(43.8)$ & 0.817 \\
\hline$>100$ & $183(51.1)$ & $109(56.5)$ & & $86(57.7)$ & $86(56.2)$ & \\
\hline \multicolumn{7}{|l|}{ CEA (ng/mL) } \\
\hline$\leq 3.4$ & $172(50.6)$ & $73(41.5)$ & 0.051 & $56(40.3)$ & $58(40.6)$ & 1.000 \\
\hline$>3.4$ & $168(49.4)$ & $103(58.5)$ & & $83(59.7)$ & $85(59.4)$ & \\
\hline \multicolumn{7}{|l|}{ WBC (cell/mm³) } \\
\hline$<3,500$ & $18(4.5)$ & $4(1.9)$ & 0.050 & $9(5.5)$ & $4(2.4)$ & 0.311 \\
\hline$\geq 3,500$ and $<10,000$ & $308(77.6)$ & $151(73.3)$ & & $120(73.2)$ & $119(72.6)$ & \\
\hline$\geq 10,000$ & 71 (17.9) & $51(24.8)$ & & 35 (21.3) & $41(25.0)$ & \\
\hline \multicolumn{7}{|l|}{ Platelet $\left(\times 10^{3} / \mathrm{mm}^{3}\right)$} \\
\hline$<130$ & $43(11.5)$ & $17(8.3)$ & 0.460 & $23(15.3)$ & $14(8.5)$ & 0.180 \\
\hline$\geq 130$ and $<400$ & $303(81)$ & $174(84.5)$ & & $115(76.7)$ & $136(82.9)$ & \\
\hline$\geq 400$ & $28(7.5)$ & $15(7.3)$ & & $12(8.0)$ & $14(8.5)$ & \\
\hline \multicolumn{7}{|l|}{ Albumin (mg/dL) } \\
\hline$\leq 3.5$ & $112(28.2)$ & $101(49)$ & $<0.001$ & $78(47.6)$ & $79(48.2)$ & 1.000 \\
\hline $3.5-5.2$ & $285(71.8)$ & $105(51)$ & & $86(52.4)$ & $85(51.8)$ & \\
\hline \multicolumn{7}{|l|}{ T.bilirubin (mg/dL) } \\
\hline$\leq 1.2$ & $315(79.3)$ & $104(50.5)$ & $<0.001$ & $100(61.0)$ & $88(53.7)$ & 0.219 \\
\hline$>1.2$ & $82(20.7)$ & $102(49.5)$ & & $64(39.0)$ & $76(46.3)$ & \\
\hline \multicolumn{7}{|l|}{$\operatorname{ALP}(I U / L)$} \\
\hline$\leq 80$ & $70(17.6)$ & 31 (15) & 0.490 & $22(13.4)$ & $25(15.2)$ & 0.753 \\
\hline$>80$ & $327(82.4)$ & $175(85)$ & & $142(86.6)$ & $139(84.8)$ & \\
\hline \multicolumn{7}{|l|}{ AST (IU/L) } \\
\hline$\leq 40$ & $275(69.3)$ & $100(48.5)$ & $<0.001$ & $91(55.5)$ & $86(52.4)$ & 0.658 \\
\hline$>40$ & $122(30.7)$ & $106(51.5)$ & & $73(44.5)$ & $78(47.6)$ & \\
\hline \multicolumn{7}{|l|}{ ALT (IU/L) } \\
\hline$\leq 40$ & $291(73.3)$ & $111(53.9)$ & $<0.001$ & $105(64.0)$ & $92(56.1)$ & 0.176 \\
\hline$>40$ & $106(26.7)$ & $95(46.1)$ & & $59(36.0)$ & $72(43.9)$ & \\
\hline \multicolumn{7}{|l|}{ Origin site } \\
\hline Bile duct & & & & 119 (72.6) & $122(74.4)$ & 0.615 \\
\hline Ampulla of Vater & & & & $12(7.3)$ & $15(9.1)$ & \\
\hline Gallbladder & & & & $33(20.1)$ & $27(16.5)$ & \\
\hline
\end{tabular}

Values are presented as number (\%) or mean \pm standard deviation. ECOG, Eastern Cooperative Oncology Group; CA 19-9, cancer antigen 19-9; CEA, carcinoembryonic antigen; WBC, white blood cell; T.bilirubin, total bilirubin; ALP, alkaline phosphatase; AST, aspartate aminotransferase; ALT, alanine transaminase. 
Table 2. Overall survival of the subgroups

\begin{tabular}{|c|c|c|c|c|}
\hline \multirow{2}{*}{ Group } & \multicolumn{2}{|c|}{ Before PSM (n=604) } & \multicolumn{2}{|c|}{ After PSM (n=328) } \\
\hline & MS (mo) & p-value & MS (mo) & p-value \\
\hline \multicolumn{5}{|l|}{ All cohort } \\
\hline Chemotherapy & 15.2 & $<0.001$ & 12.0 & 0.001 \\
\hline BSC & 7.0 & & 7.5 & \\
\hline Total & 12.1 & & 9.6 & \\
\hline \multicolumn{5}{|l|}{ Locally advanced } \\
\hline Chemotherapy & 28.0 & 0.010 & 16.7 & 0.490 \\
\hline BSC & 13.4 & & 13.4 & \\
\hline Total & 23.4 & & 14.8 & \\
\hline \multicolumn{5}{|l|}{ Metastatic } \\
\hline Chemotherapy & 12.7 & $<0.001$ & 11.1 & $<0.001$ \\
\hline BSC & 6.2 & & 6.3 & \\
\hline Total & 9.6 & & 8.3 & \\
\hline \multicolumn{5}{|l|}{ ECOG 0-1 } \\
\hline Chemotherapy & 17.0 & $<0.001$ & 13.7 & 0.001 \\
\hline BSC & 8.8 & & 8.4 & \\
\hline Total & 14.2 & & 11.3 & \\
\hline \multicolumn{5}{|l|}{ ECOG 2} \\
\hline Chemotherapy & 8.4 & 0.603 & 8.4 & 0.568 \\
\hline BSC & 6.1 & & 6.3 & \\
\hline Total & 6.6 & & 6.6 & \\
\hline \multicolumn{5}{|c|}{$\mathrm{CA} 19-9 \leq 100 \mathrm{IU} / \mathrm{mL}$} \\
\hline Chemotherapy & 19.8 & 0.001 & 12.7 & 0.330 \\
\hline BSC & 10.6 & & 10.6 & \\
\hline Total & 16.2 & & 12.3 & \\
\hline \multicolumn{5}{|c|}{ CA $19-9>100 \mathrm{IU} / \mathrm{mL}$} \\
\hline Chemotherapy & 11.8 & $<0.001$ & 10.9 & 0.001 \\
\hline BSC & 6.0 & & 6.0 & \\
\hline Total & 9.1 & & 8.2 & \\
\hline \multicolumn{5}{|l|}{$\mathrm{CEA} \leq 3.4 \mathrm{ng} / \mathrm{mL}$} \\
\hline Chemotherapy & 20.9 & 0.007 & 17.1 & 0.052 \\
\hline BSC & 11.5 & & 10.6 & \\
\hline Total & 17.8 & & 12.3 & \\
\hline \multicolumn{5}{|l|}{$\mathrm{CEA}>3.4 \mathrm{ng} / \mathrm{mL}$} \\
\hline Chemotherapy & 10.2 & $<0.001$ & 9.6 & 0.037 \\
\hline BSC & 4.9 & & 5.1 & \\
\hline Total & 8.2 & & 6.7 & \\
\hline \multicolumn{5}{|l|}{ Male } \\
\hline Chemotherapy & 14.7 & $<0.001$ & 12.8 & 0.001 \\
\hline BSC & 8.2 & & 8.3 & \\
\hline Total & 12.0 & & 10.3 & \\
\hline \multicolumn{5}{|l|}{ Female } \\
\hline Chemotherapy & 16.5 & $<0.001$ & 11.3 & 0.223 \\
\hline BSC & 6.0 & & 6.0 & \\
\hline Total & 12.4 & & 8.6 & \\
\hline
\end{tabular}

PSM, propensity score matched; MS, median survival; BSC, best supportive care; ECOG, Eastern Cooperative Oncology Group; CA 19-9, cancer antigen 19-9; CEA, carcinoembryonic antigen. 
The continuous variables were reported as the mean \pm standard deviation (SD) and compared using Student's t test. The categorical variables were described as percentages and were compared using chi-square tests.

\section{PSM and statistical analysis}

Our study was not a randomized, multicenter study, so the decision of whether to administer frontline chemotherapy in our study cohort was based on clinician discretion and patient preference. To overcome this selection bias, we performed PSM; the propensity score was calculated from a logistic regression model, and for PSM, seven baseline clinical and laboratory metrics including age (years), performance status (ECOG), CEA level, WBC level, albumin level, total bilirubin level, and AST level, were selected. With the propensity score estimated, 164 pairs of patients were matched using a 1:1 nearest neighbor matching algorithm. All statistical analyses were performed using SPSS for Windows ver. 21.0 (IBM Corp., Armonk, NY) and StataCorp. 2015, Stata Statistical Software: Release 14 (College Station, TX). Survival information was collected by the National Health Insurance Service, median survival was estimated using the Kaplan-Meier method, and comparisons between the groups were performed using the log-rank test. A p-value of less than 0.05 was considered statistically significant.

\section{Ethical statement}

This study was conducted in Korea, and Institutional Review Board approval was obtained. The study was a retrospective database review without any intervention or use of human specimens, and the requirement for informed consent was waived.

\section{Results}

\section{Patient characteristics}

A total of 604 patients with advanced BTC met our inclusion criteria; 206 were treated with only BSC without any curative treatments (chemotherapy, surgery, and radiation therapy) until their death, and 398 were treated with frontline chemotherapy.

In the pre-PSM cohort, the age of the patients was significantly older in the BSC group than in the chemotherapy group (mean $\pm S D, 60.7 \pm 10$ years vs. $69.9 \pm 10.6$ years; $p<$ 0.001). The BSC group also had poor performance statuses

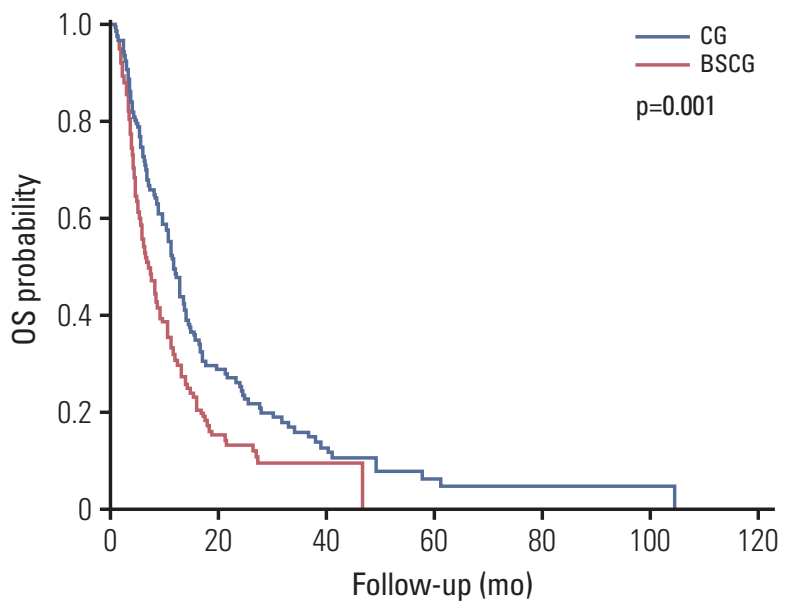

Fig. 1. All cohort (after propensity score matching analysis). CG, chemotherapy group; BSCG, best supportive care group; OS, overall survival.

and high rates of abnormal laboratory findings (albumin, total bilirubin, AST, and ALT levels) compared with the chemotherapy group. No significant differences were observed in other variables between both groups.

A total of 328 patients were matched and baseline characteristics were balanced and matched, with the exception of some laboratory variables, between the two groups. The baseline characteristics of the enrolled patients before and after PSM are listed in Table 1.

\section{Comparison of survival outcomes}

In the pre-PSM cohort, the median overall survival (OS) was 15.2 months versus 7.0 months for all cohorts in the chemotherapy group versus the BSC group. In the subgroup analysis, OS was significantly longer in the chemotherapy arm of most subgroups, except for the subgroup with an ECOG score of 2 (8.4 months for the chemotherapy group vs. 6.1 months for the BSC group, $\mathrm{p}=0.603$ ) (detailed results are shown in Table 2).

In the PSM cohort, the median OS was 12.0 months in patients given frontline chemotherapy versus 7.5 months in patients receiving BSC for all cohorts (Fig. 1). In the subgroups of patients with locally advanced disease, ECOG 2, female, CA $19-9 \leq 100 \mathrm{IU} / \mathrm{mL}$, or CEA $\leq 3.4 \mathrm{ng} / \mathrm{mL}$ there were no significant differences in OS between the chemotherapy group and the BSC group (16.7 months vs. 13.4 months [ $\mathrm{p}=0.490], 8.4$ months vs. 6.3 months [ $\mathrm{p}=0.568], 11.3$ months vs. 6.0 months $[\mathrm{p}=0.223], 12.7$ months vs. 10.6 months [ $\mathrm{p}=0.330$ ], and 17.1 months vs. 10.6 months $[\mathrm{p}=0.052]$, respectively). In the rest of the subgroups, frontline chemotherapy 

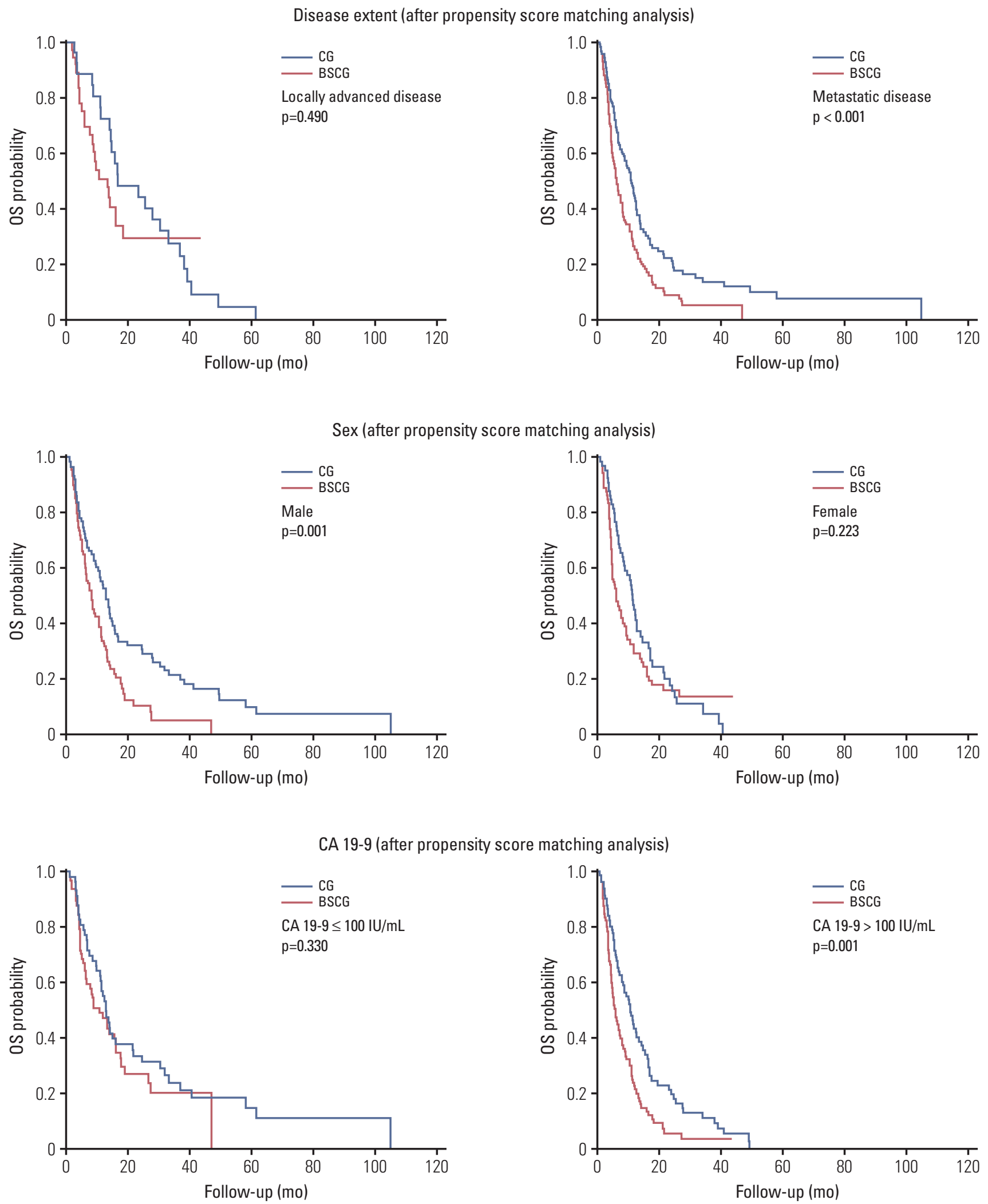

Fig. 2. Subgroup cohort (after propensity score matching analysis). CG, chemotherapy group; BSCG, best supportive care group; OS, overall survival; CA 19-9, cancer antigen 19-9; CEA, carcinoembryonic antigen; ECOG, Eastern Cooperative Oncology Group. (Continued to the next page) 
CEA (after propensity score matching analysis)
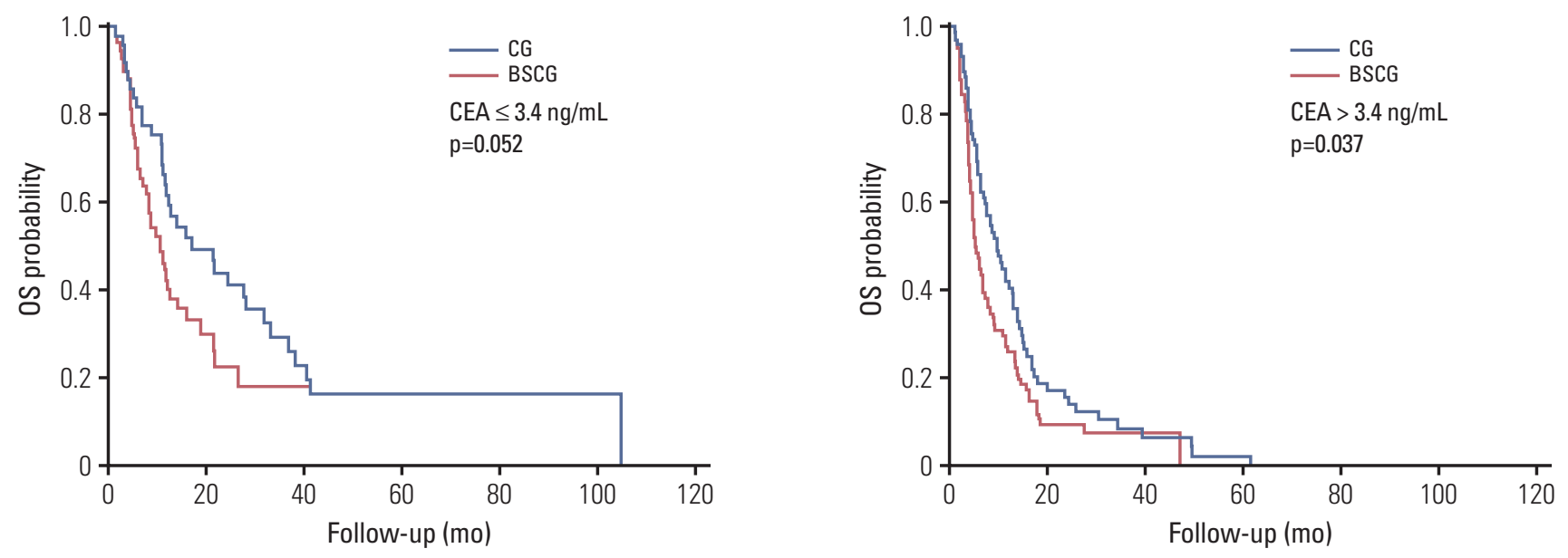

ECOG (after propensity score matching analysis)
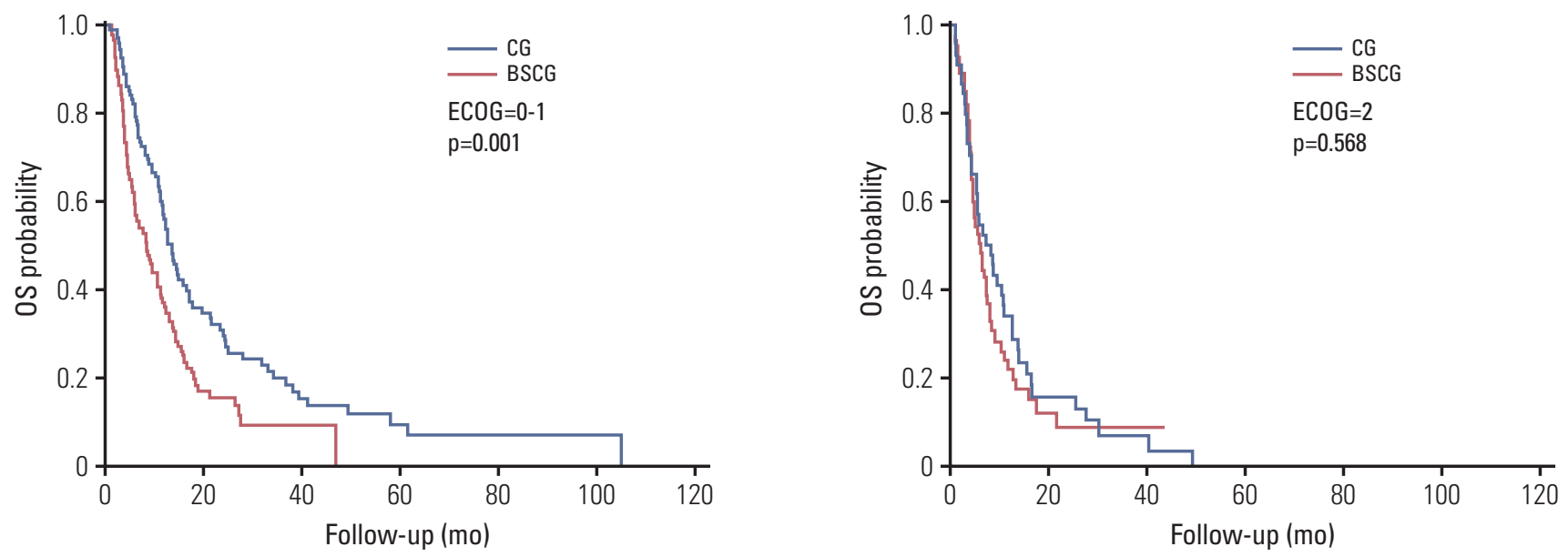

Fig. 2. (Continued from the previous page)

demonstrated a significant survival benefit (Table 2, Fig. 2). Survival analysis by the disease location revealed that there were no significant survival differences in ampulla of Vater cancer (chemotherapy group of 12.7 months vs. BSC group of 12.9 months, $\mathrm{p}=0.481$ ). But frontline chemotherapy is associated with longer survival in cholangiocarcinoma (chemotherapy group of 12.8 months vs. BSC group of 7.1 months, $\mathrm{p}=0.003$ ) and gallbladder cancer (chemotherapy group of 11.1 months vs. BSC group of 4.2 months, $\mathrm{p}=0.022$ ).

Further analyses were performed in the intersectional cohorts that consisted of subgroups with minor differences between the chemotherapy group and the BSC group. In the intersectional cohort with subgroups of locally advanced disease and CA 19-9 $\leq 100 \mathrm{IU} / \mathrm{mL} /$ locally advanced disease and $\mathrm{CEA} \leq 3.4 \mathrm{ng} / \mathrm{mL} / \mathrm{CA} 19-9 \leq 100 \mathrm{IU} / \mathrm{mL}$ and $\mathrm{CEA} \leq 3.4$ $\mathrm{ng} / \mathrm{mL}$, there were consistently no significant differences in survival between the chemotherapy group and the BSC group (23.6 months vs. 23.8 months [ $\mathrm{p}=0.509$ ], 27.1 months vs. 26.9 months [ $\mathrm{p}=0.575$ ], and 39.1 months vs. 18.8 months [ $\mathrm{p}=0.325]$, respectively) (Fig. 3).

\section{Discussion}

Although the standard treatment of inoperable BTC is combination chemotherapy with cisplatin plus gemcitabine, the efficacy of chemotherapy in advanced BTC is poor. The current use of chemotherapy in advanced BTC was extrapo- 


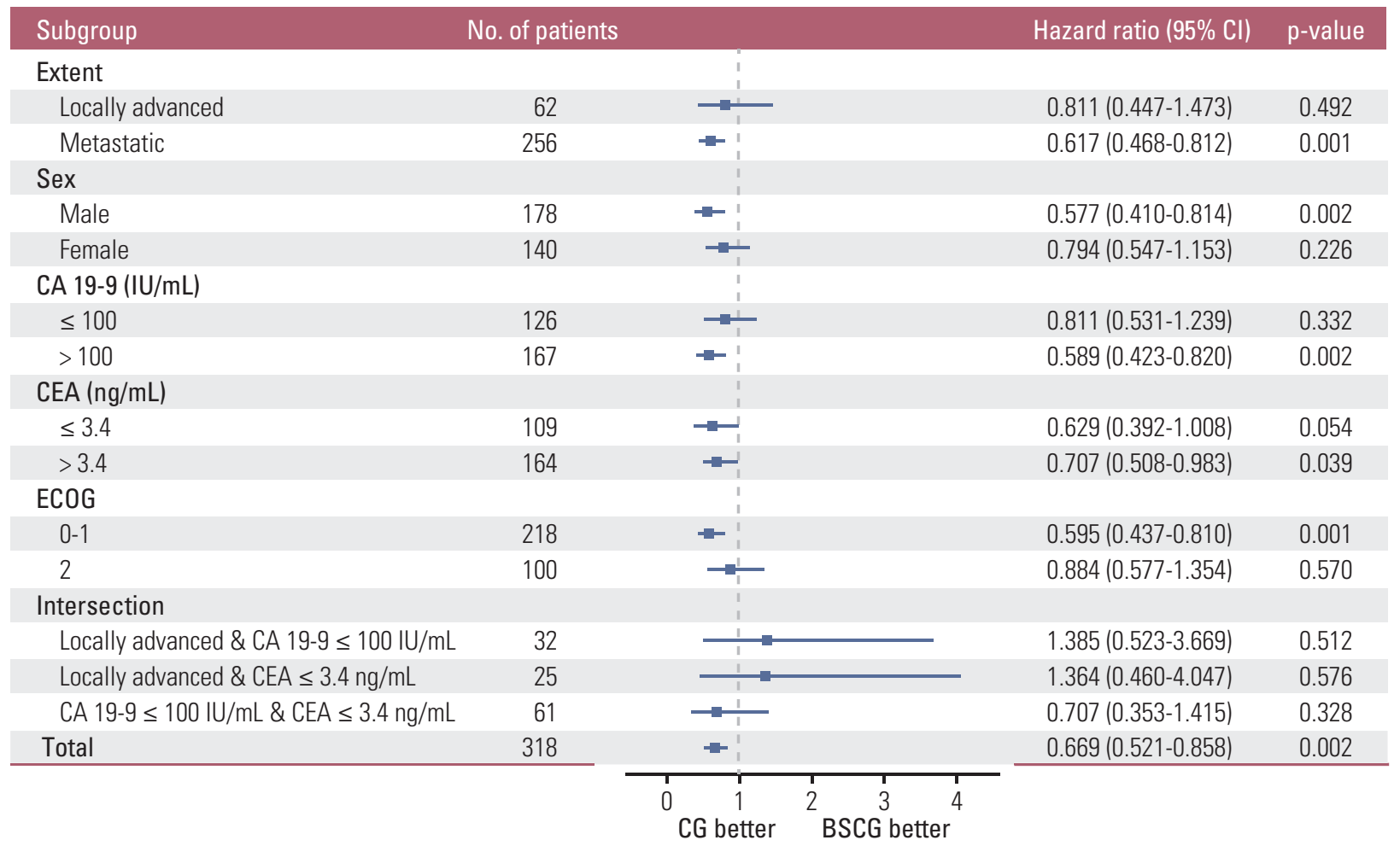

Fig. 3. Forest plot: comparison of CG versus BSCG. CI, confidence interval; CA 19-9, cancer antigen 19-9; CEA, carcinoembryonic antigen; ECOG, Eastern Cooperative Oncology Group; CG, chemotherapy group; BSCG, best supportive care group.

lated from evidence in pancreatic cancer [7]. Herein, we have shown clear evidence that chemotherapy could increase the survival rates of advanced BTC patients. Particularly, chemotherapy must be considered in cases of metastatic disease or good performance status (ECOG 0-1) or high level of tumor marker.

This study also suggests that not all patients with advanced BTC are suitable candidates for chemotherapy. There was no statistical difference between the BSC and frontline chemotherapy cohorts of advanced BTC patients with locally advanced disease and/or low levels of tumor markers. In particular, this tendency was noticeable in patients with locally advanced disease and low levels of tumor markers (CA $19-9 \leq 100 \mathrm{IU} / \mathrm{mL} / \mathrm{CEA} \leq 3.4 \mathrm{ng} / \mathrm{mL}$ ). This means that in these subgroups, immediate chemotherapy may not improve survival time. Levels of tumor markers and patient disease status are therefore important factors to consider when deciding whether or not to administer palliative chemotherapy. An additional comparative cohort study will aid in determining the optimal timing of chemotherapy; tailored treatment plans will be required for advanced BTC.

In BTC, little is known about the natural course of the disease without any cancer treatment. The majority of studies on the natural progression of BTC used a retrospective design, and a large number of the enrolled participants who were treated with only BSC already had untreatable disease with a poor performance status $[8,9]$. As such, the determined survival time of advanced BTC without any treatment is highly inaccurate and generally short. In our previous analysis of national insurance data [6], we showed that the survival time of patients with untreated advanced BTC with locally advanced disease or low levels of tumor markers (CA 19-9 $\leq 100 \mathrm{IU} / \mathrm{mL}$ or CEA $\leq 3.4 \mathrm{ng} / \mathrm{mL}$ ) was comparable to that of advanced BTC patients who were treated with cisplatin plus gemcitabine. Using the available data for the natural course of advanced BTC, a PSM analysis was used for the comparison of frontline chemotherapy and BSC. Since the first use of PSM [10], many comparative studies have been conducted using PSM when randomized trials were not possible. In the current circumstances of advanced BTC, in which cisplatin plus gemcitabine is already considered the standard therapy, a randomized comparative study is not feasible for ethical reasons. Accordingly, our PSM analysis is the proper method to compare frontline chemotherapy and BSC in advanced BTC patients with a good performance status. 
Appropriate practical guidance regarding the timing of chemotherapy initiation is lacking. Several available studies are limited to the adjuvant setting, and delayed timeto-chemotherapy may have a negative prognostic impact in breast cancer and colorectal cancer [11-14]. This trend is predominantly observed in the subgroup of patients with a poor prognosis when the remainder of the subgroup has a fair prognosis [15]. This is consistent with our results, which indicate that the prompt initiation of chemotherapy in a subgroup of advanced BTC patients with a good prognosis does not prolong survival. In the palliative setting, the generally accepted practice is that the earlier the initiation of chemotherapy the better. However, this is not always the case. A meta-analysis of data on metastatic colorectal cancer found no difference in OS, time-to-progression, or quality of life with immediate versus delayed chemotherapy [16]. There is no clear recommendation regarding the timing of chemotherapy initiation in BTC, and considering the various clinical courses of BTC, more research is necessary to better determine the optimal timing of chemotherapy in advanced BTC.

The current study has several limitations. Retrospective design is the first weak point. To reduce bias for retrospective design, we applied PSM analysis. As a stratification parameter, we selected variables by a multivariate analysis including age, extent of disease, ECOG status albumin level, total bilirubin level and AST level. Small sample size is the second weak point. The disease location is important prognostic factor but we could not include because of small sample size. Survival benefit was not observed between chemotherapy group and BSC group in specific subgroups but the result in the subgroups of $C E A \leq 3.4$ or locally advanced disease just could not satisfy statistical significance, the trend of survival was superior in chemotherapy group. If we analyzed a larger sample size, survival benefit might be favorable to the chemotherapy group. However, as we investigated credible insurance OS data and intersectional analysis, we focused on the possibility that urgent chemotherapy is not needed in some cases of BTC patient.

Actually in our previous study [6], median survival time by the disease location were ampullary cancer (11.2 months) followed by extrahepatic cholangiocarcinoma ( 9.7 months), intrahepatic cholangiocarcinoma (4.7 months), and gallbladder cancer (4.4 months). Recently, interest for quality of life (QoL) is increasing and it is as important as survival result in study for cancer. Evaluation of QoL was not performed as well in our study for the retrospective analysis.

In conclusion, our results suggest that chemotherapy in advanced BTC is mostly beneficial for survival. Especially in subgroups with metastatic disease, high tumor marker or good performance status (ECOG 0-1) this trend is evident. The other way, immediate chemotherapy may not be helpful to someone who had low tumor marker, poor performance status or localized disease. The identification of prognostic subgroups classified by their clinical and laboratory characteristics is important for optimizing the timing of the initiation of palliative chemotherapy. In addition, further genomic research to validate our results is needed, as is an additional prospective study focusing on the timeliness of chemotherapy initiation in advanced BTC.

\section{Conflicts of Interest}

Conflict of interest relevant to this article was not reported.

\section{References}

1. Huguier M, Barrier A, Valinas R, Flahault A, Adloff M, Pezet $\mathrm{D}$, et al. Randomized trial of 5-fluorouracil, leucovorin and cisplatin in advanced pancreatic cancer. Hepatogastroenterology. 2001;48:875-8.

2. Mallinson CN, Rake MO, Cocking JB, Fox CA, Cwynarski MT, Diffey BL, et al. Chemotherapy in pancreatic cancer: results of a controlled, prospective, randomised, multicentre trial. $\mathrm{Br}$ Med J. 1980;281:1589-91.

3. Palmer KR, Kerr M, Knowles G, Cull A, Carter DC, Leonard RC. Chemotherapy prolongs survival in inoperable pancreatic carcinoma. Br J Surg. 1994;81:882-5.

4. Brieau B, Dahan L, De Rycke Y, Boussaha T, Vasseur P, Tougeron $\mathrm{D}$, et al. Second-line chemotherapy for advanced biliary tract cancer after failure of the gemcitabine-platinum combination: a large multicenter study by the Association des
Gastro-Enterologues Oncologues. Cancer. 2015;121:3290-7.

5. Fornaro L, Vivaldi C, Cereda S, Leone F, Aprile G, Lonardi S, et al. Second-line chemotherapy in advanced biliary cancer progressed to first-line platinum-gemcitabine combination: a multicenter survey and pooled analysis with published data. J Exp Clin Cancer Res. 2015;34:156.

6. Ji JH, Song HN, Kim RB, Oh SY, Lim HY, Park JO, et al. Natural history of metastatic biliary tract cancer (BTC) patients with good performance status (PS) who were treated with only best supportive care (BSC). Jpn J Clin Oncol. 2015;45: 256-60.

7. Valle JW, Wasan H, Johnson P, Jones E, Dixon L, Swindell R, et al. Gemcitabine alone or in combination with cisplatin in patients with advanced or metastatic cholangiocarcinomas or other biliary tract tumours: a multicentre randomised phase 
II study: The UK ABC-01 Study. Br J Cancer. 2009;101:621-7.

8. Park J, Kim MH, Kim KP, Park DH, Moon SH, Song TJ, et al. Natural history and prognostic factors of advanced cholangiocarcinoma without surgery, chemotherapy, or radiotherapy: a large-scale observational study. Gut Liver. 2009;3:298-305.

9. Sharma A, Dwary AD, Mohanti BK, Deo SV, Pal S, Sreenivas $\mathrm{V}$, et al. Best supportive care compared with chemotherapy for unresectable gall bladder cancer: a randomized controlled study. J Clin Oncol. 2010;28:4581-6.

10. Sjolander A. Propensity scores and M-structures. Stat Med. 2009;28:1416-20.

11. Ahmann DL, Green SJ, Bisel HF, Ingle JN, Hahn RG, Lee RA, et al. An evaluation of early or delayed adjuvant chemotherapy in premenopausal patients with advances breast cancer undergoing oophorectomy: a later analysis. Am J Clin Oncol. 1982;5:355-8.

12. Yu KD, Huang S, Zhang JX, Liu GY, Shao ZM. Association between delayed initiation of adjuvant CMF or anthracycline- based chemotherapy and survival in breast cancer: a systematic review and meta-analysis. BMC Cancer. 2013;13:240.

13. Biagi JJ, Raphael MJ, Mackillop WJ, Kong W, King WD, Booth CM. Association between time to initiation of adjuvant chemotherapy and survival in colorectal cancer: a systematic review and meta-analysis. JAMA. 2011;305:2335-42.

14. Des Guetz G, Nicolas P, Perret GY, Morere JF, Uzzan B. Does delaying adjuvant chemotherapy after curative surgery for colorectal cancer impair survival? A meta-analysis. Eur J Cancer. 2010;46:1049-55.

15. Gagliato Dde M, Gonzalez-Angulo AM, Lei X, Theriault RL, Giordano SH, Valero V, et al. Clinical impact of delaying initiation of adjuvant chemotherapy in patients with breast cancer. J Clin Oncol. 2014;32:735-44.

16. Ackland SP, Jones M, Tu D, Simes J, Yuen J, Sargeant AM, et al. A meta-analysis of two randomised trials of early chemotherapy in asymptomatic metastatic colorectal cancer. Br J Cancer. 2005;93:1236-43. 